\title{
GRANULAR ASSEMBLY SIMULATION WITH CUNDALL's MODEL FOR THE DYNAMIC COLLAPSE OF THE STRUCTURAL FOUNDATION
}

\author{
By Daisuke UEMURA* and Motohiko HAKUNO**
}

\begin{abstract}
Dynamic collapse analysis of two types of structural foundation, deep and shallow, are performed by particle assemblies simulation with Cundall's model.

Nonlinear responses of the structural foundations by the horizontal force are obtained. Relationships between applied force and deformation with hysteresis and such phenomena as dilatancy and settlement of the particle assemblies ground are found not to be expected in the analysis of continuous media. A size of the ground model is of 3400 particles.

Keywords : granular assembly simulation
\end{abstract}

\section{INTRODUCTION}

Soil dynamics are classified roughly, depending on the soil handling method, into continuous and discrete mediums.

Handling the soil as a continuous body gives many results by deploying the finite element method. Many unsolved problems still left in the field of soil dynamics of discrete medium.

Soil is basically a non continuous mediuin of numerous particles. Some phenomena such as rock avalanche, debris flow and dilatancy of soil can be clarified only by considering the discrete granular assembly of soil. Therefore, the developing of a method to handle soil as a non continuous medium is necessary.

Soil handling methods, in case of non continuous medium, can be classified, according to what has been carried out in the field of soil dynamics, into three categories as follows :

1. Experiments using actual gravel and sand ${ }^{11}$,

2. Experiments using many cylinders of lead or photoelastic materials, and

3. Computer simulation ${ }^{2}$.

In the first category, it is difficult to grasp the interior state of the assembly microscopically. Also, there are several restrictions concerning the principle of similarity. The difference between those materials used in the experiment and those of the actual soil became a problem in the second category. In the third one, model can be analyzed microscopically and also macroscopically deploying various coefficients of the material such as the frictional one. Limitation of particle numbers and long computing time were the shortcomings of this method. New developments of the computer's capability overcome such

* Civil Engineer, Shimizu Construction Co., Tokyo, Japan.

** Member of JSCE, Professor, Earthquake Research Institute, University of Tokyo, Tokyo, Japan. 
obstacles.

In 1971, the Distinct Element Method (DEM) has been introduced by Cundall ${ }^{21}$. The rock medium was considered as being the aggregate of an element which is divided by many discontinuous planes.

This method analyzes the rock behavior numerically assuming that an individual rock element satisfies the equation of motion and the law of action and reaction. It is based on the use of an explicit numerical scheme in which the interaction of particles is monitored contact by contact. Cundall used this method to analyze the dynamics of two dimensional behavior of a rock or sand particle assembly.

Kiyama, Fujimura and Nishimura ${ }^{4)}$ also used Cundall's method to estimate the settlement of ground surface during the construction of a tunnel assuming that particles have a circular shape in 1983. Also, they analyzed the behavior of grain particles in a silo during their extrusion through a hole ${ }^{5}$.

Having entirely no idea about Cundall's research, the granular assembly simulation regarding the circular particles has been conducted on the static deformation problem of sand by Hakuno and Hirao, $1973^{6}$. Due to the lack of time required to compute the equations for all particles simultaneously, they could only solve a static problem without deploying an approximation such that employed by Cundall and considering only those forces of particles contact. Particles number was approximately several hundreds, but Cundall mentioned that the maximum number of particles may be limited to one thousand owing to the computer capacity. These analyses have been carried out using particle elements of similar radii and of very low velocity.

The phenomena to be analyzed with simulation under such restrictions are limited and the merit of such method is not sufficient if compared with the experimental one. Therefore, the authors have modified Cundall's method by introducing a rebound coefficient and tried the dynamic analysis of a soil model of more than 3,000 circular elements with different radii. Using circular elements saves also the CPU time. Accordingly, such phenomena as soil behaviors during the penetration of a pile into the ground and a non-linear response of a structural foundation under horizontal exciting load could be analyzed successfully by using this method ${ }^{7}$.

\section{DISTINCT ELEMENT METHOD BY CUNDALL}

a) Distinct Element Method

Cundall's Distinct Element Method (DEM) is based on the assumption that each element satisfies the equation of motion and that the transmission of force between the elements follows the law of action and reaction. This method is new approach to analyze the dynamic behavior of granular assemblies numerically. In early stage of the application of his method, Cundall assumed that rock medium is an assembly of polygonal elements. It took a long time for computation due to the complicated judgement of the contact between particles. Therefore, the total number of elements for the simulation was restricted by the limitation of CPU time. Later, he introduced the circular elements instead of the polygonal ones ${ }^{4)}$. In this case, the shape of each element is only determined by its radius. This differs from the case of a polygonal element. Therefore, it is easy to judge the contacts between elements and accordingly the computing time is minimized. The Authors adopted the circular elements in order to increase the number of elements used in the simulation.

The motion of a particle having a mass $m_{i}$ and the moment of inertia $I_{i}$ is expressed as follows :

$$
\begin{aligned}
& m_{i} \frac{d^{2} \boldsymbol{U}}{d t^{2}}+C_{i} \frac{d \boldsymbol{U}}{d t}+F_{i}=0 \\
& I_{i} \frac{d^{2} \varphi}{d t^{2}}+D_{i} \frac{d \varphi}{d t}+M_{i}=0 \cdots
\end{aligned}
$$

where $F_{i}$ is the sum of all forces acting on the particle and

$M_{i}$ is the sum of all moments acting on it.

$C_{i}$ and $D_{i}$ are the damping coefficients. 
$U$ is the displacement vector and $\varphi$ is the angular displacement.

Those who would like to know the details of Cundall's method, had better see the refferred papers ${ }^{22) .37 .5)}$. An elastic spring between particles should be introduced in order to estimate the force, which has been produced by other contacting particles, acting on a certain particle. The acting force can't be estimated using the law of equilibrium of force only. Particles deformation should also be considered in case of three or more contacting particles.

b) Modification of Cundall's DEM

The authors modified a little the above mentioned Cundall's method in the following point.

1) The authors neglected the viscous dashpot effect between particles. They treated graveles or rocks without clay, so they considered there are no viscous effect between particles.

2) The authors introduced a rebound coefficient at particles collision instead of the dashpot in order to dissipate the internal kinetic energy in the particles assembly. A matter resembles the reality.

\section{SOIL BEHAVIOR SURROUNDING A DEEP FOUNDATION PENETRATING INTO THE GROUND}

\section{a) Setting of Models}

Models of the ground assembly is made by falling particles into a box as shown in Fig. 1. The numerical simulation were conducted in two dimension and the used data of the simulation is indicated in Table 1.

b) Practically when a structure penetrates into the soil, soil particles move. Many studies have been conducted regarding such penetration in a plastic continuous domain. The plastic discontinuous domain hasn't been considered so much. Studies on this kind of problems have not been performed because such movement of soil particles cann't be handled through the analysis conducted for a conventional continuous medium. In the present study, a simulation was performed considering the soil as an assembly of soil particles related to a deep foundation penetrating into the ground.

c) Simulation Results

Monotonously increasing vertical forces are applied to a deep foundation. Several typical examples of locations, velocity distributions and normal force distribution of particles are shown in Fig. 2 (a) (d). The deep foundation is $9.0 \mathrm{~m}$ wide, $100 \mathrm{~m}$ long. Penetration process into ground are shown in Fig. 2 .

d) Inferences

In velocity distributions of Fig. 2, it is characteristic that only some particles just below the pile have some motions with different velocities. This agrees with the natural phenomenon of a pile with a similar sharp end while penetrating the ground. It indicates that the slipping line appears right. below the structure. Generally, soil response differs according to the site conditions and the ways of loading. For example, particles of the ground surface close to the pile float up sometimes in spite of the fact that most of them go down with the pile.

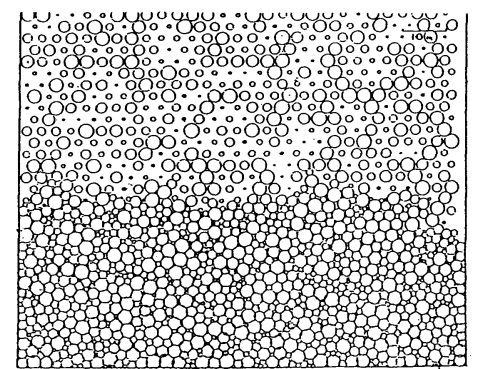

Fig. 1 Process to make particle assemblies by falling.
Table 1 Data adopted in the numerical simulation.

\begin{tabular}{|l|l|}
\hline Particle Diameter & $\begin{array}{l}0.5 \sim 5 \text { (m) } \\
\text { (uniform distribution) }\end{array}$ \\
\hline Number of particles & 3400 \\
\hline Coefficient of static friction & 0.5 \\
\hline Coefficient of dynamic friction & 0.2 \\
\hline Coefficient of rebound & 0.1 \\
\hline Cohesive force & 0 \\
\hline
\end{tabular}



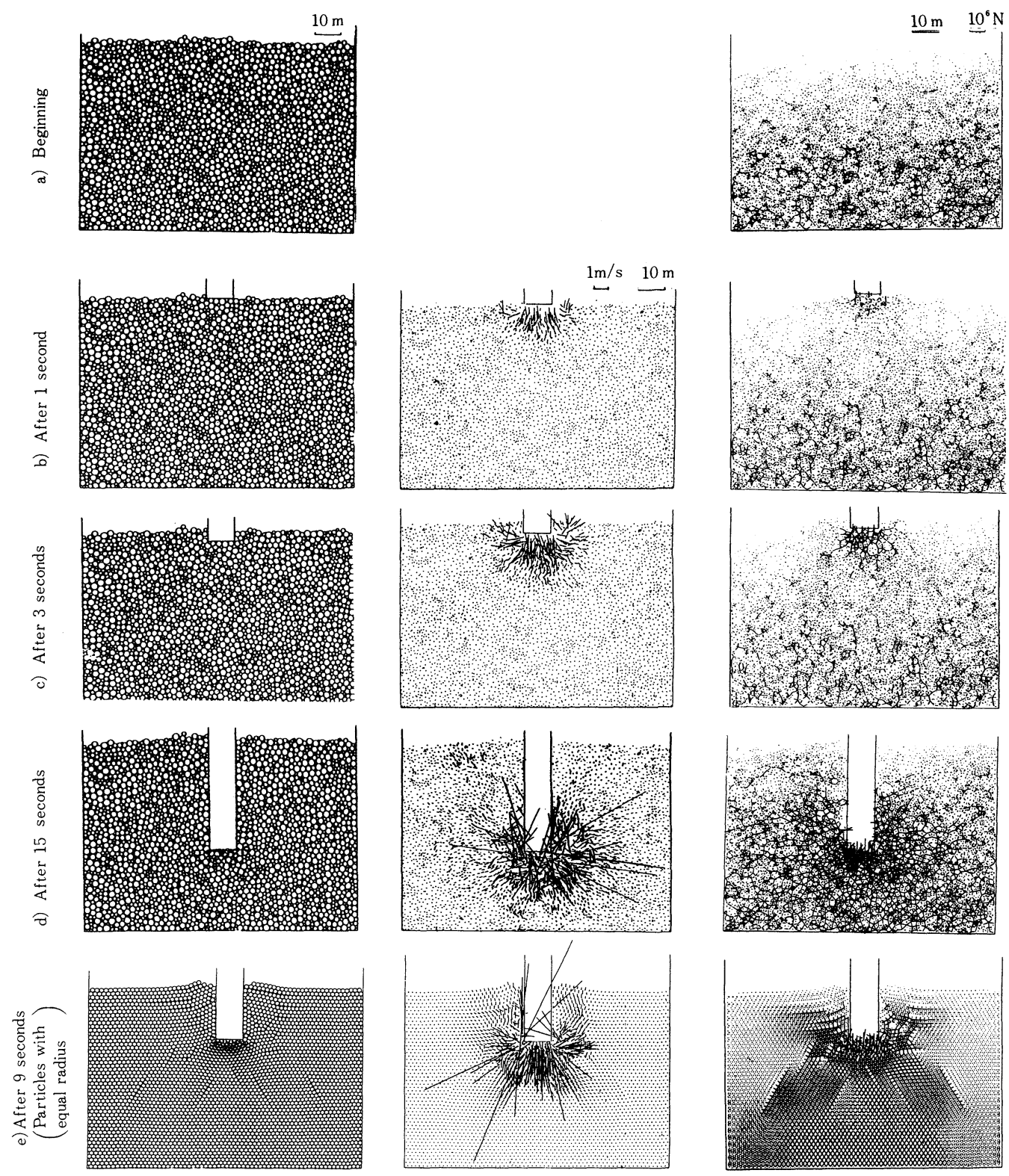

Fig. 2 Penetration of deep foundation into ground.

\section{DYNAMIC NON-LINEAR RESPONSE OF STRUCTURAL FOUNDATIONS}

The non-linear properties of a structure and its surrounding soil shoud be taken into consideration while analyzing the response of that structure under the loading conditions of earthquakes, etc. The movements of individual soil particles can not be cleared by means of the conventional continuum mechanics. The granular assembly simulation might be suitable for individual particles behavior analysis.

The behavior of a deep and a direct foundation under different horizontal dynamic loadings has been analyzed using also the previous model.

a) Model 


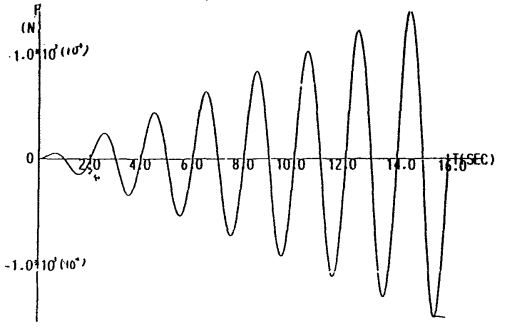

Fig. 3(a) Applied horizontal force $(0.5 \mathrm{~Hz})$ in case of $1-\mathrm{A}$ or $2-\mathrm{A}$.

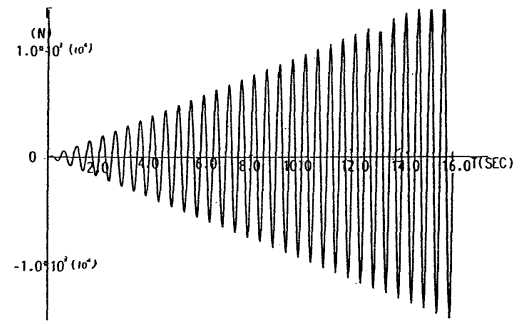

Fig. 3(b) Applied horizortal force $(2.0 \mathrm{~Hz})$ in case of $1-\mathrm{B}$ or $2-\mathrm{B}$.

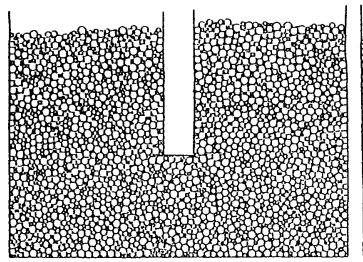

(c)

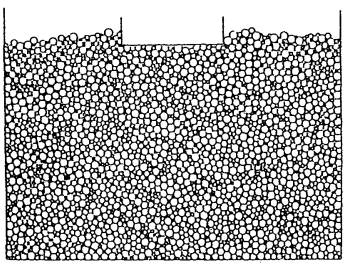

(d)
Fig. 3 Foundation model before loading.
Table 2

\begin{tabular}{|c|c|c|}
\hline $\begin{array}{c}\text { Case number } \\
\text { of numerical } \\
\text { analysis }\end{array}$ & $\begin{array}{c}\text { Type of Input Force } \\
\text { (Unit }: \mathrm{N})\end{array}$ & $\begin{array}{c}\text { Type of } \\
\text { Foun- } \\
\text { dation }\end{array}$ \\
\hline $1-\mathrm{A}$ & $\mathrm{F}=5.0 \times 10^{3} * \mathrm{t} * \sin (\pi \mathrm{t})$ & $\begin{array}{c}\text { Deep } \\
\text { Foun- } \\
\text { dation }\end{array}$ \\
\hline $1-\mathrm{B}$ & $\mathrm{F}="$ " " $(4 \pi \mathrm{t})$ & " \\
\hline $2-\mathrm{A}$ & $\mathrm{F}=5.0 \times 10^{4} * \mathrm{t} * \sin (\pi \mathrm{t})$ & $\begin{array}{l}\text { Flat } \\
\text { Foun- } \\
\text { dation }\end{array}$ \\
\hline $2-\mathrm{B}$ & $\mathrm{F}="$ " " $(4 \pi \mathrm{t})$ & " \\
\hline
\end{tabular}

The analysis was carried out for the four cases mentioned in Table 2. Dynamic loads (Fig. 3 (a) and (b) ) were applied to a deep foundation buried deeply in the ground and a flat foundation which is $30 \mathrm{~m}$ wide, $30 \mathrm{~m}$ long and five metres deep, as shown in Fig. 3 (c) and (d), (cases 1-A and 1-B). The model was simulated by releasing the concentrated stress right under the pile obtained by the penetration simulation.

Loading positions are at the pile's top and at a point five meters below the top of the flat foundation. Other assumptions were made so that both types of foundations were permitted to rotate around their bases center.

The analysis of the rotational response of foundations was carried out using the sum of particles moment, the moment of the dead weight of the foundation itself and the moments of the external forces.

b) Results of Simulation

Fig. 4 shows the location, velocity and normal force of particles for the deep foundation corresponding to approximately $0.1,0.2,0.3$, and $0.4 \mathrm{rad}$. after long dynamic excitation. Fig. 5 shows the same as Fig. 4 for the flat foundation.

The simulation results of time history of the angle and moment of a foundation are given in Fig. 6 and a small vibration response up to $2.0 \times 10^{-2}$ rad shows non-linear properties. A foundation of $10 \mathrm{~m}$ depth will be displaced up to $10 \mathrm{~cm}$ displacement of the ground surface depending on the force. Fig. 7 shows hysteresis loops (the relationship between the moment to the foundation and the angular response) utilizing data of Fig. 6. Fig. 9 and 10 indicates the loci of particles related to case 1-A and 1-B. Twelve particles have been chosen as shown in Fig. 8, and the locus of each particle has been detected.

c) Inferences

Fig. 9 and 10 show that the higher the frequency of external force, the more complicated the motion of each particle. Such motion resembles a motion of same external force's frequency together with a lower frequency motion. Individual particles mostly subsided during the vibration but they float up in some cases. The amplitude of the angular response of the foundation is greater when the frequency of external 


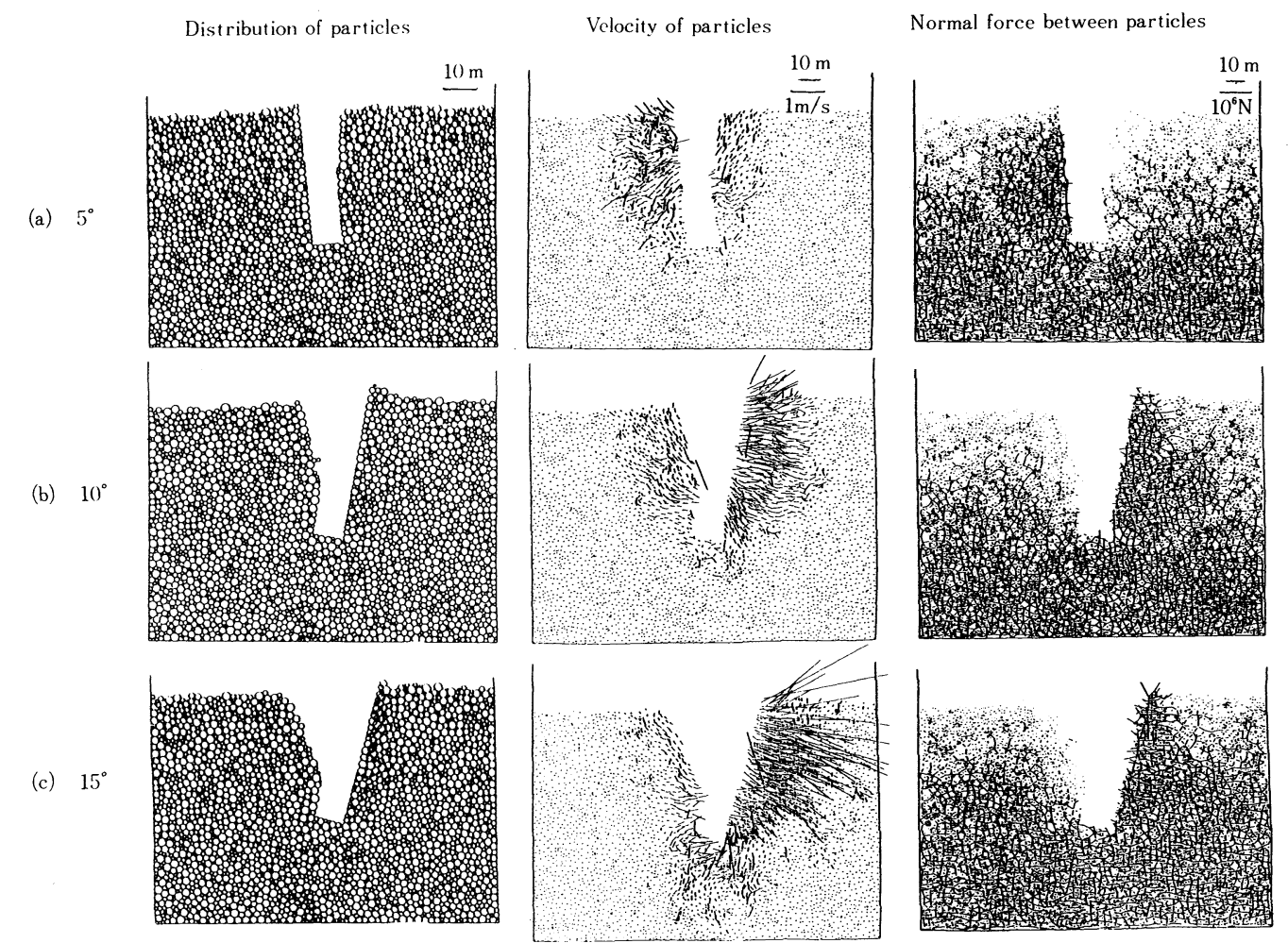

Fig. 4 Angular deformation of deep foundation by horizontal dynamic loading.

Distribution of particles

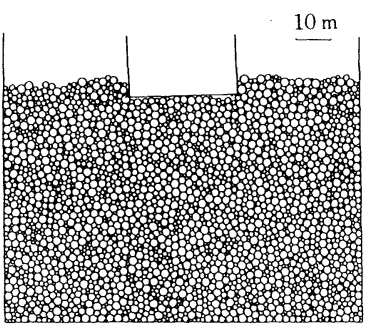

(b) $10^{\circ}$

(c) $15^{\circ}$
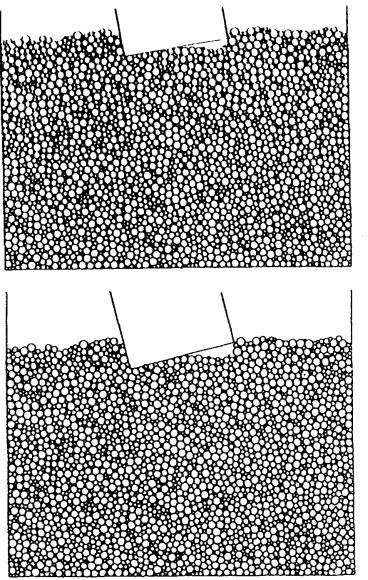

Velocity of particles
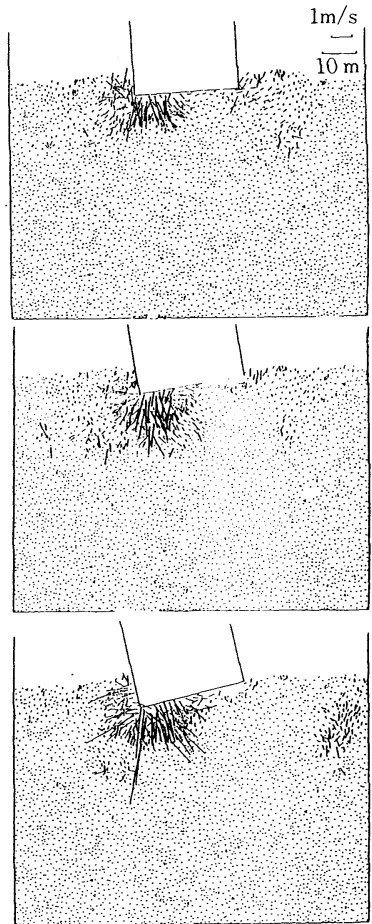

Normal force between particles
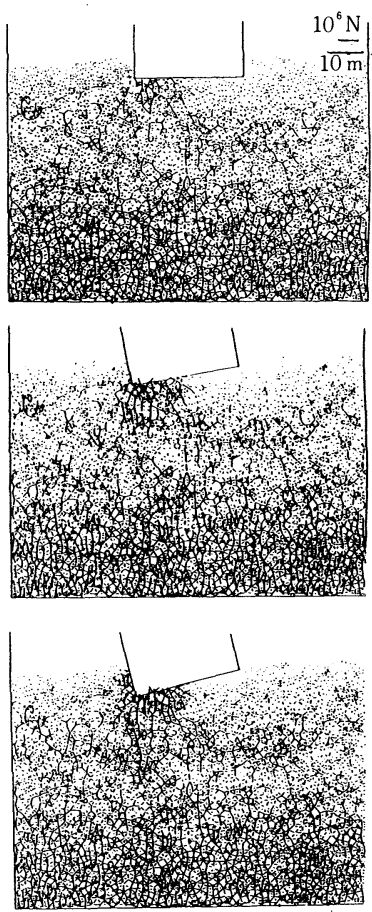

Fig. 5 Angular deformation of flat foundation by horizontal dynamic loading (Continued). 

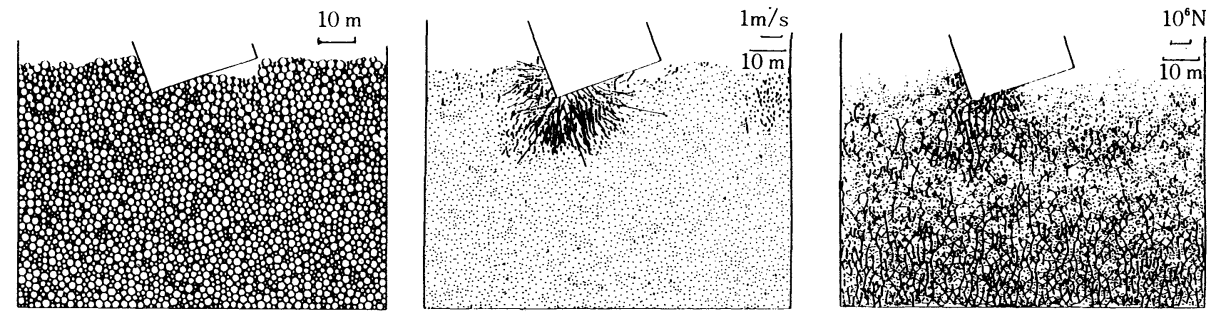

(e) $25^{\circ}$
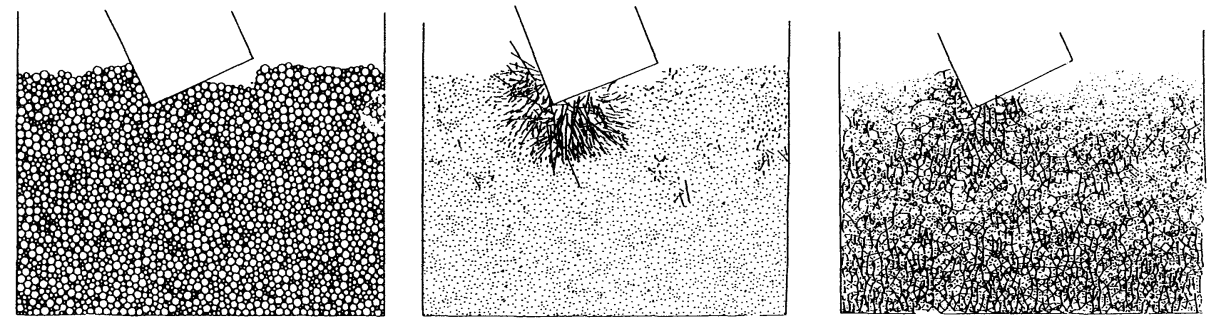

Fig. 5 (Continued).

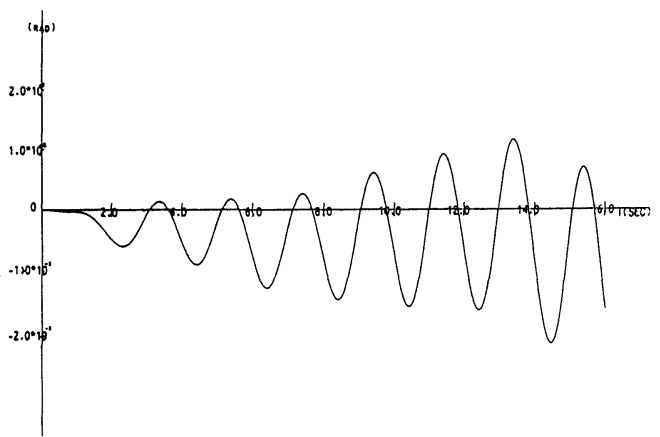

(a) Angular response of deep foundation due to $0.5 \mathrm{~Hz}$ excitation

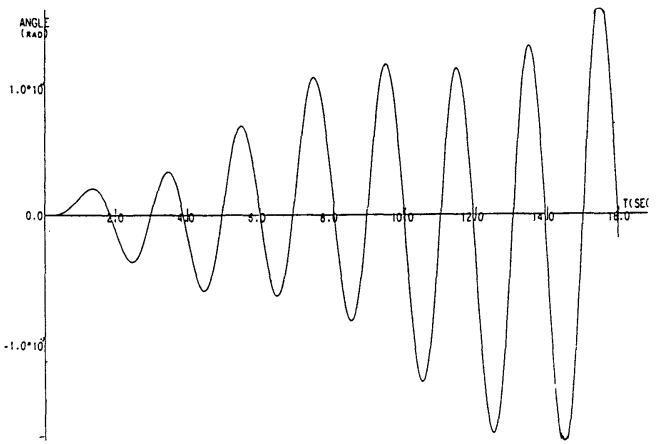

(c) Angular response of flat foundation due to $0.5 \mathrm{~Hz}$ excitation

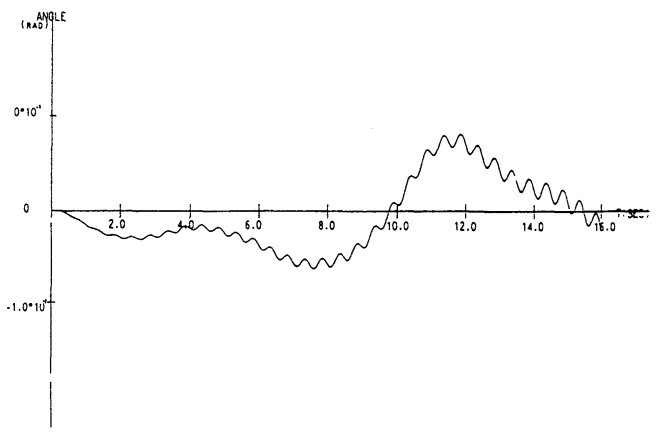

(b) Deep foundation, $2 \mathrm{~Hz}$

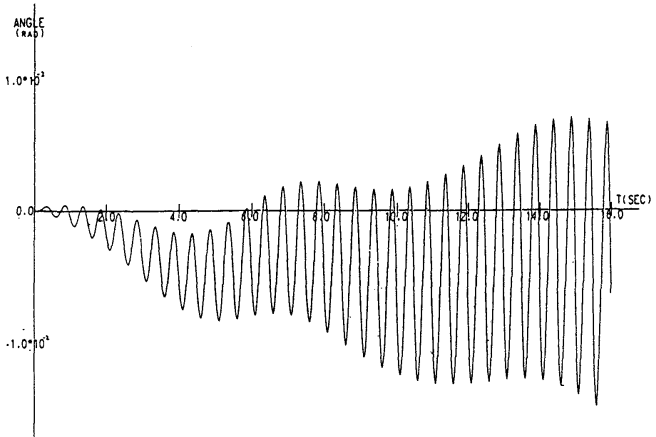

(d) Flat foundation, $2 \mathrm{~Hz}$

Fig. 6

force is $1 \mathrm{~Hz}$ than when being $4 \mathrm{~Hz}$ even if it has the same amplitude. The external force of lower frequency gives more energy to the foundation in one cycle of the vibration which might cause this phenomenon. In Fig. 7(a to d), a linear relationship is clearly defined between the angular response of the foundation and the external force when the later is small. When the external force becomes greater, the area of a hysteresis loop becomes bigger and the spring constant becomes smaller. Then, non-linear relationships 


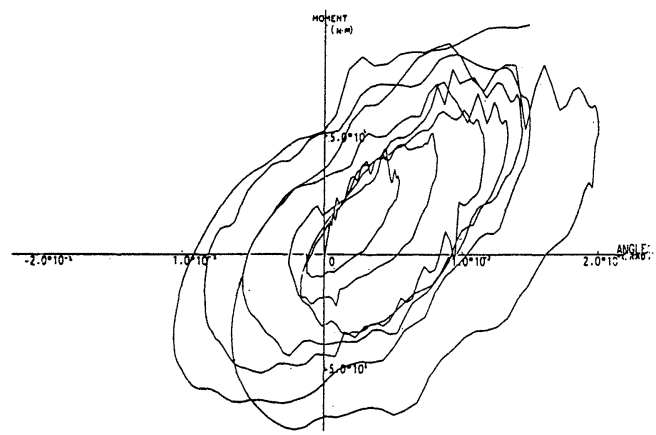

(a) Relationships between applied moment and response angle (Deep foundation, $0.5 \mathrm{~Hz}$ )

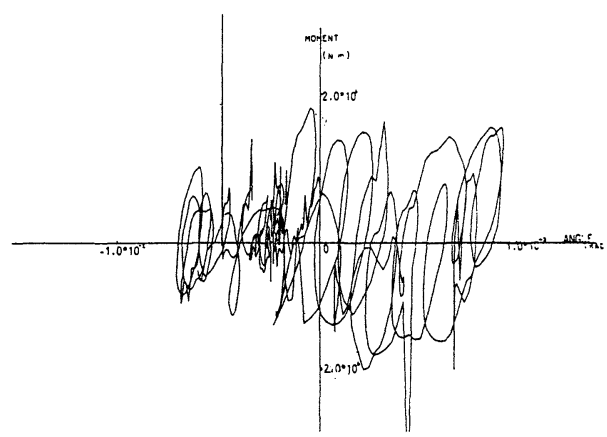

(c) Relationships between applied moment and response angle (Flat foundation, $0.5 \mathrm{~Hz}$ )

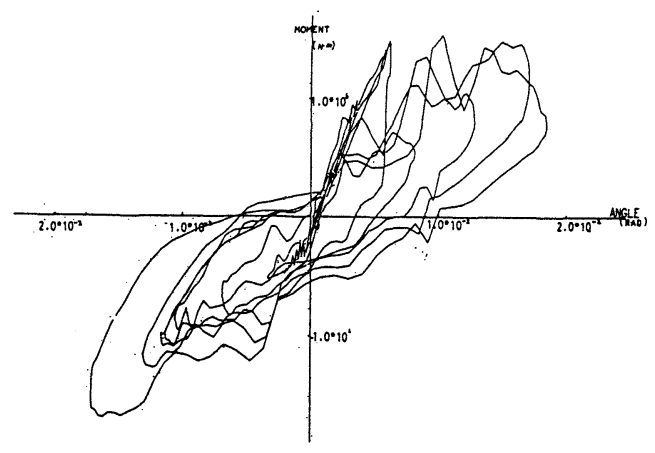

(b) Deep foundation, $2 \mathrm{~Hz}$

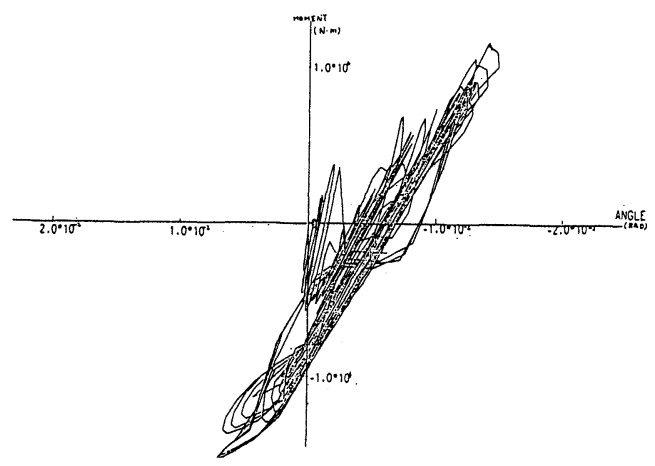

(d) Flat foundation, $2 \mathrm{~Hz}$

Fig. 7

are defined. This trend is especially well seen in the case of flat foundaion.

The relationship of case 1-B is quite different from the others. This might be caused by the large deviation of the angular response's neutral axis due to large plastic deformation.

In case 2, the spring comes to be softer in the relationships between the angular response and the high frequency external force but the area of the hysteresis loop stays almost zero for large amplitude of the external force.

The subsidence due to vibration occurred as a whole in

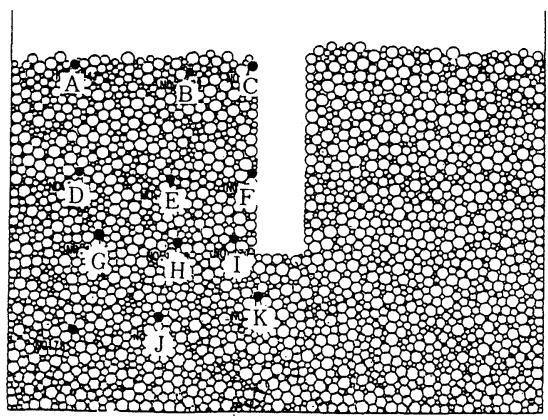

Fig. 8 Particles to detect the trace during vibration of the foundation. Fig. 9 and 10. The subsidence is larger in places close to the foundation and to the ground surface. The results of the flat foundation show that an overturn collapse of a direct foundation under a strong earthquake motion is caused due to the continuous increase of the angular response in one direction. At critical angle of inclination, the moment of the foundation's weight acts to accelerate the overturning. This is the reverse direction of the restoring force.

The results of the above mentioned simulation led to the following:

1. The non-linear relationship between the external force and the response of the foundation, obtained by field tests, was confirmed by this simulation. It is significant that these non-linear relationships were derived only from particles coefficients such as the coefficient of friction, the elastic spring constant, etc.

2. The overturning process of flat foundation was analyzed qualitatively. The foundation structure 
A

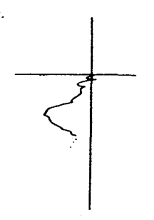

B

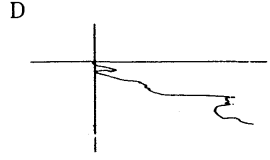

E

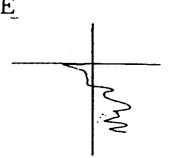

C

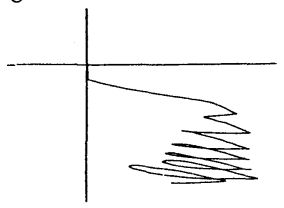

F

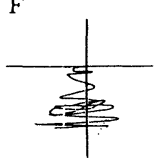

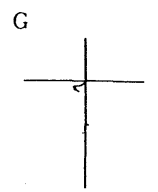

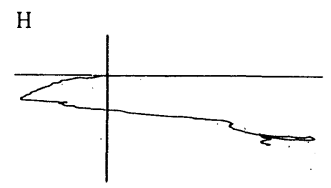

I

$J$

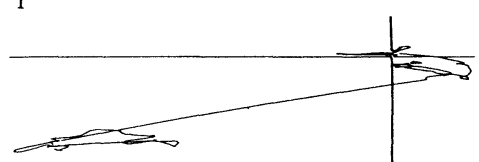

K.
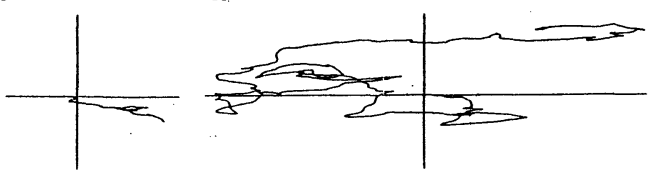

Fig. 9 Trace of each particle in Fig. 8 during vibration $(0.5 \mathrm{~Hz})$.

A

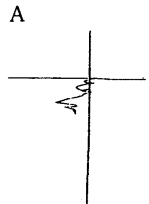

$\mathrm{D}$

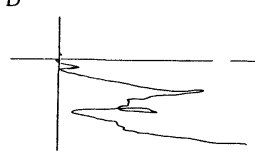

G
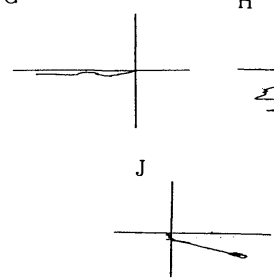

$\mathrm{H}$

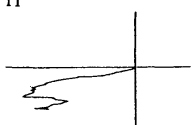

E
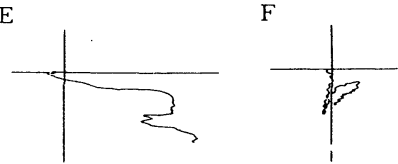

I

K

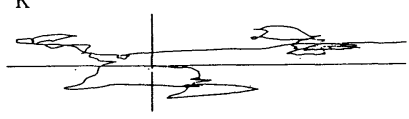

Fig. 10 Trace of each particle in Fig. 8 during vibration $(2.0 \mathrm{~Hz})$.

stop vibrating back and forth as the dead weight of the structure serves to increase the inclination angle. When the vibration amplitude becomes larger to a certain extent, the structure overturns on one side.

\section{CONCLUSION}

The authors modified Cundall's DEM (Distinct Element Method) and introduced new interpretations. Several simulations related to penetration of a pile into the ground soil and dynamic properties of a structural foundation were successfuly performed using the modified DEM. They hope this method will be 
usefull to analyze the static and dynamic problems in the structure-ground system.

\section{ACKNOWLEDGEMENTS}

We are very grateful to Mr. Masahiro Iida (Research Associate in ERI) for his helpful suggestions and continuous encouragement throughout our work. We thank Mr. Kazuyoshi Iwashita (Graduate student in ERI) very much for his kind help on the operation of the computer.

All the computations were made by the computer HITAC M280H in the computer center of the University of Tokyo.

\section{REFERENCES}

1) ASHIDA, K., EGASHIRA, S. and OHTSUKI, H. : On the flow mechanism of sliding soil from a mountain slope, Bull. Disast. Prevention Res. Inst., Kyoto Univ. No. 26-B-2, 315-327. 1982, (in Japanese)

2) CUNDALL, P. A. : A computer model for simulating progressive, large scale movements in blocky rock system, Symp. ISRM, Nancy, France, Proc. 2, 129-136, 1971.

3) CUNDALL, P. A and STRACK, O. D. L. : A discrete numerical model for granular assemblies, Geotechnique 29, 1, 47-65, 1979.

4) KIYAMA, H. , FUJIMURA, H. and NISHIMURA, T. : Analysis on the settlement of ground surface owing to tunnel excavation with Cundall model, Anual meeting of Japan Society of Civil Engineers, 3, 309-310, 1982, (in Japanese).

5) KIYAMA, H. and FUJIMURA, H. : Analysis on gravity flow of rock granular assemblies by Cundall model, Proc. Japan Society of Civil Engineer's, 333, 137-146. 1983, (in Japanese).

6) HAKUNO, M. and HIRAO, T. : A trial related to randow packing of particle assemblies, Proc. Japan Society of Civil Engineers, 219, 55-63. 1973, (in Japanese).

7) UEMURA, D. and HAKUNO, M. : Granular assembly simulation for the ground collapse, Bulettin of Earthquake Research Institute, Univ. of Tokyo, Vol. 62 No. 1, 1987, (to be published).

(Recieved May 23 1986) 